Andrade, E, Sarmento, MIA, Pereira Jr. EB, Moreira, JN, Nóbrega, EP, Caiana, CRA, Bezerra Neto, FC, Medeiros, AC \& Maracajá, PB. (2020). Reuse of water for the production of seedlings of pine cone. Research, Society and Development, 9(7):1-17, e536974403.

\title{
Reutilização de água para produção de mudas de pinha
}

\section{Reuse of water for the production of seedlings of pine cone}

Reutilización del agua para la producción de plántulas de pinares

Recebido: 10/05/2020 | Revisado: 12/05/2020 | Aceito: 17/05/2020 | Publicado: 26/05/2020

Edvanildo de Andrade

ORCID: https://orcid.org/0000-0002-3385-6599

Universidade Federal de Campina Grande, Brasil

E-mail: edvanildo@hotmail.com

Maria Iza de Arruda Sarmento

ORCID: https://orcid.org/0000-0001-8764-8114 Universidade Federal Rural do Pernambuco, Brasil

E-mail: izasarmento1@gmail.com

Ednaldo Barbosa Pereira Junior

ORCID: https://orcid.org/0000-0003-0098-3206

Instituto Federal de Educação Ciência e Tecnologia da Paraíba, Brasil

E-mail: ebprj2@hotmail.com

Joserlan Nonato Moreira

ORCID: https://orcid.org/0000-0002-2290-7119 Instituto Federal de Educação Ciência e Tecnologia da Paraíba, Brasil

E-mail: joserlan.moreira@ifpb.edu.br

Éverson Pedrosa da Nóbrega

ORCID: https://orcid.org/0000-0002-5518-9547

Instituto Federal de Educação Ciência e Tecnologia da Paraíba, Brasil

E-mail: everson_pedrosa@hotmail.com

Clarice Ribeiro Alves Caiana

ORCID: https://orcid.org/0000-0002-5374-1617

Universidade Federal de Campina Grande, Brasil

E-mail: clariceribeirocaiana@gmail.com 
Francisco das Chagas Bezerra Neto

ORCID: https://orcid.org/0000-0001-6232-4383

Universidade Federal de Campina Grande, Brasil

E-mail: chagasneto237@gmail.com

Aline Carla de Medeiros

ORCID: https://orcid.org/0000-0002-0161-3541

Universidade Federal de Campina Grande, Brasil

E-mail: alinecarla.edu@gmail.com

Patrício Borges Maracajá

ORCID: https://orcid.org/0000-0003-4812-0389

Universidade Federal da Paraíba, Brasil

E-mail: patriciomaracaja@gmail.com

\section{Resumo}

O presente trabalho objetivou avaliar o aproveitamento de água de ar condicionado e efluente agroindustrial na produção e desenvolvimento de mudas de pinha. A pesquisa foi desenvolvida no setor de produção de mudas do Instituto Federal da Paraíba, Campus Sousa. O delineamento utilizado foi o inteiramente casualizados, com quatro tratamentos e quatro repetições. Os tratamentos foram compostos de quatro proporções de água: $\mathrm{T} 1=100 \%$ de Poço Artesiano; $\mathrm{T} 2=100 \%$ de Ar condicionado; $\mathrm{T} 3=50 \%$ Ar condicionado $+50 \%$ Efluente agroindustrial e; T4 = 100\% Efluente agroindustrial. As mudas de pinha foram produzidas em sacos de polietileno, utilizando como substrato solo e esterco bovino na proporção (2:1 v.v). Foram avaliadas as seguintes variáveis: número de folhas (NF), diâmetro do colmo (DC), matéria seca da parte aérea e da raiz e a influência nos atributos do solo como o potencial de hidrogeniônico $(\mathrm{pH})$, fósforo $(\mathrm{P})$, potássio $(\mathrm{K}+)$, sódio $(\mathrm{Na}+)$, cálcio $(\mathrm{Ca}+2)$, magnésio $(\mathrm{Mg}+2)$, Soma de bases (SB), Percentual de sódio trocável (PST) e matéria orgânica (M.O). Os tratamentos T1 e T2 são alternativas viáveis para a utilização na irrigação de mudas de pinheira. O tratamento T4 não é recomendável para irrigação de mudas pinheira de devido ao nível de toxidez provocado pelos elevados teores de sódio e cloreto.

Palavras-chave: Efluente; Solo; Reuso.

\footnotetext{
Abstract

This study aimed to evaluate the utilization of air conditioning and agroindustrial effluent in the production and development of pine cone seedlings. The research was developed in the
} 
seedling production sector of the Federal Institute of Paraiba, Campus Sousa. The experimental design was completely randomized, with four treatments and four replications. The treatments consisted of four water proportions: $\mathrm{T} 1=100 \%$ of artesian well; T2 $=100 \%$ of air conditioning; $\mathrm{T} 3=50 \%$ air conditioning $+50 \%$ agroindustrial effluent; $\mathrm{T} 4=100 \%$ agroindustrial effluent. The pine cone seedlings were produced in polyethylene bags, using as soil substrate and bovine manure in proportion $(2: 1 \mathrm{v} . \mathrm{V})$. The following variables were evaluated: number of Leaves (NF), stem diameter (DC), dry matter of the aerial part and root dry and the influence on soil attributes such as the potential of hydrogenionic $(\mathrm{pH})$, phosphorus $(\mathrm{P})$, potassium $(\mathrm{K}+)$, sodium $(\mathrm{Na}+)$, calcium $(\mathrm{Ca}+2)$, Magnesium $(\mathrm{Mg}+2)$, Sum of bases (SB), percentage of exchangeable sodium (PST) and organic matter (M. O). The treatments $\mathrm{T} 1$ and $\mathrm{T} 2$ are viable alternatives for the use in irrigation of seedlings of pine cone. The T4 treatment is not recommended for irrigation of pine cone seedlings due to the toxicity level caused by the high levels of sodium and chloride.

Key words: Effluent; Soil; Reuse.

\section{Resumen}

El presente trabajo tenía por objeto evaluar el uso del agua procedente del aire acondicionado y del efluente agroindustrial en la producción y desarrollo de plántulas de cono de pino. La investigación se desarrolló en el sector de la producción de plántulas del Instituto Federal de Paraíba, Campus Sousa. El diseño fue completamente aleatorio, con cuatro tratamientos y cuatro réplicas. Los tratamientos se componían de cuatro proporciones de agua: T1 a 100\% pozo artesiano; T2 - 100\% Aire Acondicionado; T3 - 50\% Aire acondicionado + 50\% Efluentes agroindustriales y; T4 - 100\% Efluente agroindustrial. Las plántulas de pino se produjeron en bolsas de polietileno, utilizando como sustrato el suelo y el estiércol bovino en proporción (2:1 v.v). Se evaluaron las siguientes variables: número de hojas (NF), diámetro del tallo (DC), polvo y materia seca de raíz e influencia en los atributos del suelo como potencial hidrológico $(\mathrm{pH})$, fósforo $(\mathrm{P})$, potasio $(\mathrm{K}+)$, sodio $(\mathrm{Na}+)$, calcio $(\mathrm{Ca}+2)$, magnesio $(\mathrm{Mg}+2)$, Suma de bases (SB), Porcentaje de sodio intercambiable (PST) y materia orgánica (M.O). Los tratamientos T1 y T2 son alternativas viables para el uso en el riego de plántulas de pino. El tratamiento Con T4 no se recomienda para el riego de plántulas de pino debido al nivel de toxicidad causado por los altos niveles de sodio y cloruro.

Palabras clave: Efluente; Solo; Reutilización. 


\section{Introdução}

A relação de demanda e oferta de água necessita do estabelecimento de políticas adequadas e da implementação de sistemas de gestão efetivos. Diversos são os instrumentos e tecnologias a serem utilizados no tratamento dessa questão, porém, vários deles carecem de estudos que viabilizem o seu melhor emprego e produzam resultados ambientais e econômicos satisfatórios. Uma das alternativas que se tem apontado para enfrentamento do problema é a técnica de reuso de água.

A eficiência do reuso de águas residuárias na irrigação, a necessidade do desenvolvimento de pesquisas que proporcionem melhorias nessa técnica, bem como a inevitável busca por fontes alternativas de água, instiga a investigação do reuso de águas residuárias na produção de mudas, etapa fundamental na instalação de um pomar vigoroso e sadio, aspectos que refletirão na produtividade do mesmo.

A aplicação de águas residuárias na irrigação agrícola é uma alternativa viável para aumentar a disponibilidade hídrica em regiões áridas e semiáridas. Estudos apontam que a produtividade agrícola aumenta significativamente ao serem irrigadas com o uso de águas residuárias. O acúmulo de contaminantes químicos no solo é um efeito negativo que pode ocorrer dependendo das características físico-químicas da água residuária utilizada.

Com uma produção de frutas de aproximadamente 40 milhões de toneladas por ano, o Brasil ocupa o terceiro lugar na produção mundial de frutas, contudo a participação do país no comércio global do setor é de apenas $2 \%$, o que demonstra o forte consumo interno (Gazeta, 2010).

No sertão da Paraíba, o Instituto Federal da Paraíba (IFPB) Campus Sousa, vem adotando nos últimos anos, políticas de reestruturação física, instalando sistemas de refrigeração em vários espaços fechados da instituição, visando proporcionar conforto aos que frequentam estes ambientes. Tal atitude vem propiciando oportunidade de captação de água, oriundo do sistema de ar condicionado, já instalado, possibilitando o seu reuso para irrigação. Existe também na Instituição um complexo agroindustrial, do qual, diariamente são expelidos dezenas de litros de águas cinzas, que surgem como uma excelente alternativa para o estudo, visando a sua reutilização para fins de irrigação.

Com intuito de aproveitar efluente agroindustrial, água do sistema de ar condicionado e sua combinação como forma de reuso e otimização da água bruta, Bezerra et. al (2019) constataram que a água de ar condicionado é uma alternativa viável para a utilização na irrigação de plântulas de mamoeiro, sendo o efluente agroindustrial o menos recomendável 
em virtude dos elevados teores de sódio e cloreto.

O aproveitamento de efluentes domésticos ou industriais, como fonte de adubação orgânica e de água para irrigação de culturas agrícolas pode favorecer o desenvolvimento de uma determinada comunidade ou região, principalmente nos casos onde a escassez de água é o maior problema para o aumento da produção agrícola. $\mathrm{O}$ reuso da água pode proporcionar melhoramentos econômicos relacionados ao aumento da área cultivada e da produtividade decorrente da contribuição de nutrientes encontrado nestas águas, proporcionando alternativas de explorar áreas, mesmo em localidade que não existam reservatórios com água suficientemente destinada à irrigação.

Diante destes fatores, tem-se buscado métodos alternativos de reutilização da água, como o aproveitamento das águas pluviais, água-cinza, águas residuais tratadas e a dessalinização, que aparecem como meios de conservação da água e como alternativas para enfrentar a falta desse recurso, tanto para fins potáveis quanto não potáveis, tornando uma opção prática e a baixo custo para minimizar a escassez (Pushard, 2008).

Entretanto, na região em estudo ainda pouco se fala sobre o aproveitamento e reutilização de água para fins agrícolas, tornando-se necessário desenvolver este trabalho que visa analisar o desenvolvimento de mudas de uma espécie frutífera, a pinha (Annona squamosa L.), irrigada com diferentes tipos de águas residuárias, como água de ar condicionado e efluente agroindustrial.

\section{Metodologia}

O experimento foi realizado no período de março a julho de 2017 no viveiro de mudas do Instituto Federal de Educação, Ciência e Tecnologia da Paraíba (IFPB) - Campus Sousa, unidade São Gonçalo.

Durante o período do experimento, as condições climáticas do Perímetro Irrigado de São Gonçalo são caracterizadas por precipitação pluvial média de $21,8 \mathrm{~mm}$, temperatura média de $28,4^{\circ} \mathrm{C}$, o que provocou uma taxa de evaporação média de 243,35 mm. Conforme a classificação de Köppen, o clima da região é do tipo BSh, o que indica um clima semiárido quente, marcado por taxas de evapotranspiração potencial anual superior à precipitação anual e à inexistência de cursos de água permanentes (Brasil, 2018; Brasil, 2019).

Os tratamentos resultaram em T1 $=100 \%$ água de poço; $\mathrm{T} 2=100 \%$ água dos sistemas de ar condicionados; T3 = combinação $(50 \%$ de água do ar condicionado $+50 \%$ de efluente agroindustrial) e T4 $=100 \%$ efluente agroindustrial, e o delineamento experimental utilizado 
foram blocos completamente casualizados, com quatro tratamentos e quatro repetições, sendo cada parcela experimental composta por quatro plantas.

O sistema de coleta de água compõe tubos de PVC de $20 \mathrm{~mm}$ conectados na saída de cada mangueira de dreno dos aparelhos e direcionados para uma caixa coletora de 5001 no Bloco de Agroecologia, localizado no Instituto Federal da Paraíba Campus Sousa, composto por 16 dependências climatizadas, com sistema de ar condicionado que variam na faixa de 12.000 a 24.000 BTUS, sendo que sete ficam ligados diariamente no horário de expediente (Pereira Jr et al., 2018).

O efluente agroindustrial foi coletado no Bloco de Agroindústria, através de um desvio, feito na tubulação de esgoto do laboratório de Processamento de Leite e Derivados, com destino a uma caixa coletora com capacidade de 5001 (Ferreira Neto et al., 2017). A coleta da água proveniente de poço foi feita em um poço artesiano localizado próximo ao Bloco de Agroindústria;

Na produção das mudas foram usadas como materiais propagativos, sementes de pinha (Annona squamosa L.) oriundas de plantios localizados no setor de produção de fruticultura do IFPB - Campus Sousa. As mesmas foram retiradas manualmente de frutos sadios e maduros, lavadas e mantidas à sombra em local arejado para secagem durante uma semana.

As sementes foram acondicionadas em sacos de polietileno de 1,2 $\mathrm{L}$, que foram preenchidos manualmente com substrato produzido a partir de areia, barro e esterco bovino, na proporção (2:1 v.v). Uma amostra do substrato foi coletada e encaminhada ao Laboratório de Análises de Solo, Água e Plantas (LASAP), do IFPB Campus Sousa, para análise, cujos resultados encontram-se na Tabela 1 .

Tabela 1: Resultado da análise química do substrato preparado para produção de pinha UFCG, Campus Pombal/IFPB, Sousa 2017.

\begin{tabular}{|c|c|c|c|c|c|c|c|c|c|c|c|}
\hline $\mathrm{pH}$ & $\mathrm{N}$ & $\mathrm{P}$ & M.O. & K & $\mathrm{Na}$ & $\mathrm{Ca}$ & $\mathrm{Mg}$ & $\mathrm{H}+\mathrm{Al}$ & SB & CTC & V \\
\hline $\mathrm{H}_{2} \mathrm{O}$ & $\mathrm{g} / \mathrm{kg}$ & $\mathrm{mg} \mathrm{dm}^{-3}$ & $\mathrm{~g} \mathrm{~kg}^{-1}$ & --- & ---- & ------ & $\mathrm{mol}_{\mathrm{c}}$ & 3 & - & - & $\%$ \\
\hline 7,5 & 3,19 & 3,63 & 66,34 & 4,45 & 0,44 & 14,8 & 2,1 & 0,0 & 21,79 & 21,79 & 100 \\
\hline
\end{tabular}

Fonte: Autores.

Para a semeadura, adotou-se três sementes por recipiente, na profundidade de $2 \mathrm{~cm}$, cobrindo-as com uma fina camada de substrato e uma camada superficial de casca de arroz para conservar a umidade e favorecer a germinação. Após a germinação, fez-se necessário o desbaste das plântulas menos vigorosas, deixando apenas uma plântula por recipiente. Foram 
realizadas irrigações diárias (manhã e tarde), fornecendo um volume de água suficiente para elevar a umidade do substrato e mantendo-o próximo à capacidade de campo. Ao longo do período experimental foram realizadas capinas manuais.

As amostras das águas utilizadas nas irrigações também foram encaminhadas ao LASAP do IFPB Campus Sousa, para análises físico-químicas, cujos resultados encontram-se na Tabela 2.

Tabela 2: Resultado da análise química das águas utilizadas para irrigação. UFCG, Campus Pombal/IFPB, Sousa 2017.

\begin{tabular}{|c|c|c|c|c|c|c|c|c|c|c|c|c|c|c|}
\hline \multirow[b]{2}{*}{ Fonte } & $\mathrm{pH}$ & CE & K & $\mathrm{Na}$ & $\mathrm{Ca}$ & $\mathrm{Mg}$ & $\mathrm{SO}_{4}$ & $\mathrm{CO}_{3}{ }^{2-}$ & $\mathrm{HCO}_{3}^{-}$ & $\mathrm{Cl}^{-}$ & CSR & $\mathrm{NaCl}$ & $\mathrm{CaCO}_{3}$ & RAS \\
\hline & & $\mathrm{dS} \mathrm{m}^{-1}$ & -- & ------ & ---- & - & $-\mathrm{mmo}$ & ----- & -------- & ------ & & \multicolumn{2}{|c|}{---mg L L-1---- } & $\left(\mathrm{mmol}_{\mathrm{c}} \mathrm{L}\right)^{0,5}$ \\
\hline PA & 8,0 & 0,89 & 0,14 & 0,89 & 6,1 & 1,4 & 0,47 & 0,72 & 10,08 & 1,45 & 4,02 & 409,0 & 348,0 & 0,45 \\
\hline $\mathrm{AC}$ & 7,2 & 0,04 & 0,01 & 0,00 & 0,1 & 0,04 & 0,00 & 0,00 & 1,52 & 0,1 & 1,47 & 12,36 & 15,3 & 0,00 \\
\hline $\begin{array}{c}\mathrm{AC}+ \\
\mathrm{EA}\end{array}$ & 7,0 & 1,52 & 0,72 & 0,57 & 3,9 & 1,1 & 0,08 & 0,00 & 14,52 & 4,45 & 9,52 & 712,0 & 599,0 & 0,36 \\
\hline EA & 6,9 & 2,75 & 1,49 & 5,02 & 7,3 & 2,2 & 0,17 & 0,00 & 27,5 & 8,55 & 18,0 & 1313,0 & 1101,0 & 2,30 \\
\hline
\end{tabular}

$\mathrm{PA}=$ Poço Artesiano $\mathrm{AC}=\mathrm{Ar}$ condicionado $\mathrm{AC}+\mathrm{EA}=50 \%$ de água de ar condicionado $+50 \%$ de Efluente agroindustrial; EA = Efluente agroindustrial. Fonte: Autores.

Aos 60, 90 e 120 dias após a semeadura (DAS) foram avaliadas as características morfológicas: Comprimento da Parte Aérea (CPA), obtido pela distância entre a região do colo e a gema apical do ramo principal (com o auxílio de régua graduada em $\mathrm{cm}$ ), Diâmetro do Caule (DC) apenas aos 60 e 90 dias, obtido pela medição das plantas a quatro centímetros acima do colo (com o auxílio de paquímetro digital) e número de folhas (NF), obtido pela contagem total do número de folhas totalmente expandidas.

Na última avaliação aos 120 dias determinou-se a massa seca da parte aérea (MFPA) e massa seca da raiz (MFR), para isso, se fez necessário separar a raiz do caule da planta, as mesmas foram acondicionadas separadamente em sacos de papel, etiquetadas e secas em estufa de circulação forçada de ar a $65{ }^{\circ} \mathrm{C}$, permitindo a circulação forçada de ar até peso constante, todos estes procedimentos foram realizados no LASAP do IFPB - Campus Sousa.

Ao final do experimento foram coletados amostras de solo em cada parcela experimental para determinação do $\mathrm{pH}$ e os teores de fósforo $(\mathrm{P})$, potássio $(\mathrm{K})$, sódio $(\mathrm{Na}+)$, cálcio $(\mathrm{Ca}+)$, magnésio $(\mathrm{Mg}+)$, matéria orgânica $(\mathrm{MO})$ e porcentagem de sódio trocável (PST) com o objetivo de verificar possíveis mudanças nas características do solo durante o período experimental, analisada conforme a metodologia da EMBRAPA (1997) no LASAP do IFPB, Campus Sousa.

Os resultados foram submetidos à Análise de Variância (ANOVA) e as médias, 
comparadas pelo teste de Tukey, a 0,05 de probabilidade, através do programa computacional

- SISVAR (Ferreira, 2011).

\section{Características Morfológicas da Planta}

Houve diferença significativa em nível de $\mathrm{p} \leq 0,05$ de probabilidade para Altura de Planta aos 30, 60 e 90 dias (Tabela 3).

Tabela 3: Altura média de plantas de mudas de pinha (Annona squamosa) irrigadas com fontes alternativas de água e efluente agroindustrial. UFCG, Campus Pombal/IFPB, Sousa 2019.

\begin{tabular}{|c|c|c|c|}
\hline \multirow{3}{*}{ Tratamentos } & \multicolumn{3}{|c|}{ ALTURA DE PLANTA (cm) } \\
\hline & 30 dias & 60 dias & 90 dias \\
\hline & \multicolumn{3}{|c|}{----------Após aplicação dos tratamentos --- } \\
\hline T1 & $12,61 \mathrm{ab}$ & $16,42 \mathrm{a}$ & $31,22 \mathrm{a}$ \\
\hline $\mathrm{T} 2$ & $13,15 \mathrm{a}$ & $16,59 \mathrm{a}$ & 31,89 a \\
\hline T3 & $11,95 \mathrm{ab}$ & $14,68 \mathrm{ab}$ & $19,08 \mathrm{~b}$ \\
\hline $\mathrm{T} 4$ & $11,33 \mathrm{~b}$ & $13,76 \mathrm{~b}$ & $0,00 \mathrm{c}$ \\
\hline $\mathrm{CV} \%$ & 5,04 & 7,24 & 13,16 \\
\hline $\mathbf{F}$ & $*$ & $*$ & $*$ \\
\hline DMS & 1,36 & 2,45 & 5,97 \\
\hline
\end{tabular}

$\mathrm{T} 1$ = Poço Artesiano; $\mathrm{T} 2=$ Ar condicionado; $\mathrm{T} 3=50 \%$ de água de ar condicionado $+50 \%$ de Efluente agroindustrial; T4 = Efluente agroindustrial. Médias seguidas, em linha, com letras diferentes apresentaram efeitos significativo em nível de $(\mathrm{p}<0,05)$ pelo teste Tukey. $\mathrm{CV}=$ Coeficiente de Variação. DMS = Diferença Mínima Significativa. Médias seguidas pela mesma letra na coluna, não diferem entre si, pelo teste de Tukey. * $=(\mathrm{p} \leq 0,05)$ de probabilidade. Fonte: Autores.

Aos 30 dias, o tratamento T2 apresentou o melhor resultado para a variável altura de planta, enquanto que aos 60 e 90 dias os tratamentos T1 e T2 apresentaram os melhores resultados para a variável estudada.

Os tratamentos T3 e T4 apresentaram ao longo dos 90 dias os menores valores de altura de planta, o que pode estar diretamente relacionado com os níveis de salinidade presentes no tratamento aplicado às plantas. Considerando-se que o aumento da concentração de sais no solo acarreta prejuízos no desenvolvimento e crescimento das plantas (Sá et al., 2013), principalmente quando essas concentrações têm o sódio como sal mais representativo, como observados nas plantas que receberam tratamento com os maiores níveis de salinidade (Sá et al., 2015).

Quanto a variável diâmetro médio do caule (Tabela 4), os tratamentos T1 e T2 estudados apresentaram diferença média significativa $(\mathrm{p} \leq 0,05)$ aos 60 e 90 dias. Estes 
Research, Society and Development, v. 9, n. 7, e536974403, 2020

(CC BY 4.0) | ISSN 2525-3409 | DOI: http://dx.doi.org/10.33448/rsd-v9i7.4403

apresentaram aos 60 e 90 dias os maiores valores, mostrando-se serem indicadas para uso na produção de mudas de pinheira.

Tabela 4: Diâmetro Médio do Caule de mudas de pinha (Annona squamosa) irrigadas com fontes alternativas de água e efluente agroindustrial. UFCG, Campus Pombal/IFPB, Sousa 2019.

\begin{tabular}{ccc}
\hline \multicolumn{2}{c}{ DIÂMETRO DO CAULE $(\mathbf{m m})$} & \\
\hline & \multicolumn{6}{c}{$\mathbf{6 0}$ dias } & $\mathbf{9 0}$ dias \\
\cline { 2 - 3 } Tratamentos & ----- Após aplicação dos tratamentos----- \\
\hline T1 & $3,16 \mathrm{a}$ & $0,15 \mathrm{a}$ \\
T2 & $3,17 \mathrm{a}$ & $0,15 \mathrm{a}$ \\
T3 & $2,83 \mathrm{~b}$ & $0,11 \mathrm{~b}$ \\
T4 & $2,62 \mathrm{~b}$ & $0,00 \mathrm{c}$ \\
\hline CV \% & 3,77 & 9,01 \\
F & $*$ & $*$ \\
DMS & 0,24 & 0,02 \\
\hline
\end{tabular}

$\mathrm{T} 1$ = Poço Artesiano; $\mathrm{T} 2=$ Ar condicionado; $\mathrm{T} 3=50 \%$ de água de ar condicionado $+50 \%$ de Efluente agroindustrial; T4 = Efluente agroindustrial. Médias seguidas, em linha, com letras diferentes apresentaram efeitos significativos em nível de $(\mathrm{p}<0,05)$ pelo teste Tukey. CV = Coeficiente de Variação. DMS = Diferença Mínima Significativa. Médias seguidas pela mesma letra na coluna, não diferem entre si, pelo teste de Tukey. * $=(\mathrm{p} \leq 0,05)$ de probabilidade. Fonte: Autores.

Aos 60 dias os tratamentos T3 e o T4, não diferiram estatisticamente entre si, embora o tratamento com efluente agroindustrial fosse reduzido, não chegando aos 90 dias, provavelmente devido às mudanças morfológicas em condição de estresse salino, também observado por Silva (1997) e Santos (2000), constatando decréscimo na emissão foliar em gravioleira, com aumento do nível de salinidade.

Quanto à variável Número de Folhas (Tabela 5), observa-se que aos 30 dias após a aplicação dos tratamentos, as plantas que receberam aplicação de água de Poço artesiano, Ar condicionado e 50\% de água de ar condicionado $+50 \%$ de efluente agroindustrial não apresentaram diferença significativa $(\mathrm{p} \leq 0,05)$, destacando-se como os melhores valores para a variável em estudo. 
Tabela 5: Número Médio de Folhas de mudas de pinha (Annona squamosa) irrigadas com fontes alternativas de água e efluente agroindustrial. UFCG, Campus Pombal/IFPB, Sousa 2019.

\begin{tabular}{|c|c|c|c|}
\hline \multirow{3}{*}{ Tratamentos } & \multicolumn{3}{|c|}{ NÚMERO DE FOLHAS } \\
\hline & 30 dias & 60 dias & 90 dias \\
\hline & \multicolumn{3}{|c|}{-------------Após aplicação dos tratamentos ---------- } \\
\hline T1 & $7,75 \mathrm{a}$ & $12,50 \mathrm{ab}$ & $19,50 \mathrm{a}$ \\
\hline $\mathrm{T} 2$ & $7,75 \mathrm{a}$ & $13,00 \mathrm{a}$ & $19,50 \mathrm{a}$ \\
\hline $\mathrm{T} 3$ & $6,50 \mathrm{ab}$ & $9,50 \mathrm{~b}$ & $10,50 \mathrm{~b}$ \\
\hline $\mathrm{T} 4$ & $5,75 \mathrm{~b}$ & $5,00 \mathrm{c}$ & $0,00 \mathrm{c}$ \\
\hline $\mathrm{CV} \%$ & 9,68 & 13,99 & 17,09 \\
\hline $\mathbf{F}$ & $*$ & $*$ & $*$ \\
\hline DMS & 1,48 & 3,09 & 4,67 \\
\hline
\end{tabular}

$\mathrm{T} 1=$ Poço Artesiano; $\mathrm{T} 2=$ Ar condicionado; $\mathrm{T} 3=50 \%$ de água de ar condicionado $+50 \%$ de Efluente agroindustrial; T4 = Efluente agroindustrial. Médias seguidas, em linha, com letras diferentes apresentaram efeitos significativo em nível de $(\mathrm{p}<0,05)$ pelo teste Tukey. CV = Coeficiente de Variação. DMS = Diferença Mínima Significativa. Médias seguidas pela mesma letra na coluna, não diferem entre si, pelo teste de Tukey. * $=(\mathrm{p} \leq 0,05)$ de probabilidade. Fonte: Autores.

Aos 60 e 90 dias após a aplicação dos tratamentos, têm se observado comportamento semelhante para os tratamentos T1 e T2. Enquanto o tratamento T4 apresentou redução do número médio de folhas, chegando a $100 \%$ aos 90 dias. De acordo com Chartzoulakis e Klapaki (2000), esse comportamento decorre do aumento da salinidade no substrato, o que reduz a absorção de água pelas raízes, inibindo a atividade meristemática e o alongamento celular, tendo como consequência a redução no crescimento e desenvolvimento da cultura.

Quanto aos valores de Massa seca da Parte Aérea (Tabela 6), observa-se que os tratamentos que receberam Água de Poço Artesiano e água de Ar Condicionado apresentaram os resultados satisfatórios, diferindo estatisticamente $(\mathrm{p} \leq 0,05)$ dos demais tratamentos aplicados.

Já para a variável Massa Seca da Raiz ainda na Tabela 6, os melhores resultados foram observados nos tratamentos que receberam água de Poço Artesiano, água oriunda de Ar condicionado e 50\% de água de ar condicionado $+50 \%$ de efluente agroindustrial, sendo estes os tratamentos que apresentam em sua composição química as menores relações de absorção de sódios (RAS), 0,45, 0,00 e 0,36 $\mathrm{mmol}^{-0,5}$ respectivamente, comparadas com 2,30 $\mathrm{mmol}^{-0,5}$ do tratamento que recebeu apenas efluente agroindustrial (Tabela 2), sendo este um valor acima do tolerado pela a maioria das culturas, o que provavelmente ocasionou a morte das plantas que receberam esse tratamento. 
Tabela 6: Valores médios de massa seca da parte érea e de raiz de mudas de pinha (Annona squamosa) irrigadas com fontes alternativas de água e efluente agroindustrial. UFCG, Campus Pombal/IFPB, Sousa 2019.

\begin{tabular}{ccc}
\hline Tratamentos & \multicolumn{2}{c}{ Massa Seca } \\
\cline { 2 - 3 } & Parte Aérea & Raiz \\
\hline T1 & $13,02 \mathrm{a}$ & $11,59 \mathrm{a}$ \\
T2 & $14,21 \mathrm{a}$ & $11,81 \mathrm{a}$ \\
T3 & $8,22 \mathrm{~b}$ & $9,77 \mathrm{a}$ \\
T4 & $0,00 \mathrm{c}$ & $0,00 \mathrm{~b}$ \\
\hline CV \% & 15,49 & 14,02 \\
F & $*$ & $\mathrm{~ns}$ \\
DMS & 3,03 & 2,56 \\
\hline
\end{tabular}

$\mathrm{T} 1$ = Poço Artesiano; $\mathrm{T} 2=$ Ar condicionado; $\mathrm{T} 3=50 \%$ de água de ar condicionado $+50 \%$ de Efluente agroindustrial; T4 = Efluente agroindustrial. Médias seguidas, em linha, com letras diferentes apresentaram efeitos significativo em nível de $(\mathrm{p}<0,05)$ pelo teste Tukey. $\mathrm{CV}=$ Coeficiente de Variação. DMS = Diferença Mínima Significativa. Médias seguidas pela mesma letra na coluna, não diferem entre si, pelo teste de Tukey. * $=(\mathrm{p} \leq 0,05)$ de probabilidade. Fonte: Autores.

Testando fontes e proporção de água e efluente agroindustrial Nóbrega et al. (2017) Constatou que o uso da água de efluente agroindustrial inferiu menores resultados para matéria verde e seca da parte aérea no crescimento inicial de mudas de goiabeira, de maracujazeiro (Lima et al., 2019) e de mamoeiro (Bezerra, et al., 2019) nas mesmas condições experimentais.

As reduções na massa seca das plantas com aumento da salinidade da água também foram observadas por Távora, Lima e Hernandez (2004), estudando a gravioleira, e por Gurgel et al. (2007) com a aceroleira, sendo estas reduções atribuídas aos efeitos osmóticos e iônicos ocasionados pela salinidade (Sá et al., 2015).

Nóbrega et al. (2017), testando efluente agroindustrial constatou necrose nas mudas de goiabeira aos 120 dias após a semeadura provocado pelo efeito tóxico do cloreto e sódio.

\section{Influência dos Atributos Químicos do Solo}

Não houve diferença significativa para os teores de $\mathrm{pH}, \mathrm{P}$ e $\mathrm{Ca}^{+2}$ (tabela 7) e M.O (Tabela 8) ao contrário do $\mathrm{K}^{+2}$ e $\mathrm{Na}^{+2}$ (Tabela 7) e $\mathrm{Mg}^{+2,}$ SB CTC e PST (tabela 8) apresentaram efeitos significativos entre os tratamentos em estudo.

$\mathrm{O}$ pH do solo permaneceram próximo da neutralidade evidenciando que os tratamentos não interferiram durante o período experimental (Tabela 7). Enquanto que Bezerra et al. (2019) testando diferentes tipos de água na produção de mudas de mamoeiro constatou o pH alcalino no solo ao final do experimento. 
Tabela 7: Valores médios de potencial Hidrogeniônico $(\mathrm{pH})$, Fósforo disponível $(\mathrm{P})$, Potássio (K), Sódio $\left(\mathrm{Na}^{+}\right)$e Cálcio $\left(\mathrm{Ca}^{+2}\right)$ em amostras de solo após produção de mudas de pinha. UFCG, Campus Pombal/IFPB, Sousa 2019.

\begin{tabular}{cccccc}
\hline Tratamentos & $\mathbf{p H}$ & $\mathbf{P}$ & $\mathbf{K}^{+}$ & $\mathbf{C a}^{+\mathbf{2}}$ & $\mathbf{N a}^{+}$ \\
\cline { 2 - 6 } & & $\mathrm{mg} \mathrm{dm}{ }^{-3}$ & \multicolumn{2}{c}{$---\mathrm{cmol}_{\mathrm{c}} \mathrm{dm}^{-3}$------- } \\
\hline $\mathrm{T} 1$ & $7,70 \mathrm{a}$ & $4,08 \mathrm{a}$ & $2,49 \mathrm{c}$ & $11,05 \mathrm{a}$ & $1,25 \mathrm{c}$ \\
$\mathrm{T} 2$ & $7,52 \mathrm{a}$ & $4,38 \mathrm{a}$ & $2,90 \mathrm{bc}$ & $12,05 \mathrm{a}$ & $0,62 \mathrm{c}$ \\
$\mathrm{T} 3$ & $7,62 \mathrm{a}$ & $3,91 \mathrm{a}$ & $3,61 \mathrm{ab}$ & $12,85 \mathrm{a}$ & $3,18 \mathrm{~b}$ \\
$\mathrm{~T} 4$ & $7,57 \mathrm{a}$ & $4,13 \mathrm{a}$ & $4,20 \mathrm{a}$ & $13,45 \mathrm{a}$ & $5,48 \mathrm{a}$ \\
\hline CV\% & 1,16 & 9,46 & 11,58 & 11,60 & 18,41 \\
F & $\mathrm{ns}$ & $\mathrm{Ns}$ & $*$ & $\mathrm{~ns}$ & $*$ \\
DMS & 0,19 & 0,86 & 0,84 & 3,16 & 1,07 \\
\hline
\end{tabular}

$\mathrm{T} 1=$ Poço Artesiano; $\mathrm{T} 2=$ Ar condicionado; $\mathrm{T} 3=50 \%$ de água de ar condicionado $+50 \%$ de Efluente agroindustrial; $\mathrm{T} 4=$ Efluente agroindustrial. $\quad \mathrm{CV}=$ Coeficiente de Variação. DMS $=$ Diferença Mínima Significativa. Médias seguidas pela mesma letra na coluna, não diferem entre si, pelo teste de Tukey. ${ }^{*}=$ $(p \leq 0,05)$ de probabilidade. Fonte: Autores.

Segundo Ayers \& Westcot (1991), do pH nas águas a serem utilizadas para irrigação recomendam que o valor do $\mathrm{pH}$ se encontre entre 6,5 a 8,4. A concentração $\mathrm{H}+\mathrm{e} \mathrm{OH}-$, contida nas águas de irrigação, pode exercer influência na disponibilidade e absorção de nutrientes por parte das plantas, na estrutura e propriedades do solo e nos sistemas de irrigação Duarte et al. (2008).

As concentrações de fósforo (P) no solo permaneceram baixas (tabela 7), praticamente não ocorreram alterações comparadas aos teores de $\mathrm{P}$ antes da condução do experimento (tabela 1). Esses dados corroboram com Bezerra et al. (2019) que na produção de mudas de mamoeiro irrigando com diferentes tipos de água observou também não significância nos teores baixos de $\mathrm{P}$ no solo no final do período experimental.

Quanto aos valores de Potássio $\left(\mathrm{K}^{+}\right)$e Cálcio $\left(\mathrm{Ca}^{+2}\right)$ na tabela 7 , observa-se que apesar da adição do nutriente através da aplicação dos tratamentos, nota-se que após aplicação dos tratamentos o solo apresenta valores menores de $\mathrm{K}^{+}$comparados ao valor inicial $\left(4,45 \mathrm{cmol}_{\mathrm{c}}\right.$ $\mathrm{dm}^{-3}$ ), o que pode ser atribuído a absorção pelas plantas de pinheira, tendo em vista que são macronutrientes, juntamente com nitrogênio e fósforo, mais requeridos entre as anonáceas (Marchal \& Bertin, 1980).

Para valores de Sódio $\left(\mathrm{Na}^{+}\right)$, nota-se que o tratamento que recebeu apenas efluente agroindustrial apresentou os maiores valores, decorrente dos valores de $\mathrm{Na}^{+}$presentes na composição do efluente agroindustrial.

Segundo (Herpin et al., 2007; Leal et al., 2009) também constataram aumento nos teores de $\mathrm{Na}^{+}$no solo quando utilizaram efluente tratado de origem doméstica, atribuiu esse acúmulo pelo fato de o teor desse elemento no efluente ser elevado. 
Para os valores de Magnésio $\left(\mathrm{Mg}^{+2}\right)$ (Tabela 8), observa-se diferença significativa $(\mathrm{p} \leq 0,05)$ entre os tratamentos avaliados, de forma que os tratamentos T1 e T2 apresentaram os melhores valores, embora os tratamentos T3 e T4 não diferiram dos dois primeiros tratamentos.

Tabela 8: Valores médios de Magnésio $\left(\mathrm{Mg}^{+2}\right)$, Matéria Orgânica (MO), Porcentagem de Sódio Trocável (PST), Soma de Bases (SB) e Capacidade de Troca Catiônica (CTC).

\begin{tabular}{cccccc}
\hline Tratamentos & $\mathbf{M g}^{+2}$ & MO & PST & SB & CTC \\
\cline { 2 - 6 } & $\mathrm{cmol}_{\mathrm{c}} \mathrm{dm}^{-3}$ & $\mathrm{~g} \mathrm{~kg}^{-1}$ & $\%$ & $---\mathrm{cmol}_{\mathrm{c}} \mathrm{dm}^{-3}----$ \\
\hline $\mathrm{T} 1$ & $5,05 \mathrm{a}$ & $70,84 \mathrm{a}$ & $6,25 \mathrm{c}$ & $19,84 \mathrm{~b}$ & $19,84 \mathrm{~b}$ \\
$\mathrm{~T} 2$ & $2,25 \mathrm{~b}$ & $66,18 \mathrm{a}$ & $3,75 \mathrm{c}$ & $17,83 \mathrm{~b}$ & $17,83 \mathrm{~b}$ \\
$\mathrm{~T} 3$ & $3,35 \mathrm{ab}$ & $64,79 \mathrm{a}$ & $13,50 \mathrm{~b}$ & $23,60 \mathrm{a}$ & $23,60 \mathrm{a}$ \\
$\mathrm{T} 4$ & $3,55 \mathrm{ab}$ & $66,20 \mathrm{a}$ & $21,25 \mathrm{a}$ & $26,09 \mathrm{a}$ & $26,09 \mathrm{a}$ \\
\hline CV\% & 25,23 & 5,82 & 22,21 & 5,92 & 5,92 \\
F & $*$ & $\mathrm{~ns}$ & $*$ & $*$ & $*$ \\
DMS & 1,97 & 8,60 & 5,48 & 2,85 & 2,85 \\
\hline
\end{tabular}

$\mathrm{T} 1$ = Poço Artesiano; $\mathrm{T} 2=$ Ar condicionado; $\mathrm{T} 3=50 \%$ de água de ar condicionado $+50 \%$ de Efluente agroindustrial; $\mathrm{T} 4=$ Efluente agroindustrial. $\mathrm{CV}=$ Coeficiente de Variação. DMS $=$ Diferença Mínima Significativa. Médias seguidas pela mesma letra na coluna, não diferem entre si, pelo teste de Tukey. $*=$ $(\mathrm{p} \leq 0,05)$ de probabilidade. Fonte: Autores.

Tal comportamento pode estar diretamente relacionado com os teores de Matéria Orgânica presentes no solo, Leonel et al. (2015) explica que o aumento dos teores de $\mathrm{Mg}^{+2}$ deve-se ao fato das maiores doses de matéria orgânica aportarem também maiores quantidades de magnésio no solo.

Quanto aos valores de Porcentagem de Sódios Trocáveis (PST), o maior valor foi verificado no tratamento T4, fato que pode ser explicado devido aos valores elevados de sais presentes na composição química do tratamento aplicado, mostrando que não são recomendados para uso na irrigação de produção de mudas de pinha, haja vista que a PST nesses tratamentos apresenta-se acima de 21\%, enquadrando-se como um solo sódico e inapropriado para produção de mudas de pinheira.

A utilização de água com altos níveis de $\mathrm{Na}^{+}$altera a percentagem de saturação por sódio do solo, podendo prejudicar a cultura em PST a partir de 15 a 20\%, além de ocasionar clorose, morte de folhas e diminuição da estatura de plantas (Carmona et al., 2011).

Para os valores de Soma de Bases e Capacidade de Troca Catiônica (Tabela 8), observa que os tratamentos que receberam $50 \%$ de água de ar condicionado $+50 \%$ de efluente agroindustrial apresentaram os maiores valores, estando relacionado, em parte, ao aumento da disponibilidade de $\mathrm{Na}^{+}$ao solo. Para Santana et al. (2003) observaram que a 
irrigação com água salina aumentou os valores da CTC total, efetiva, soma e saturação por bases em um solo cultivados com feijoeiro.

Consoante Santana et al. (2003), os menores valores da soma de bases nos tratamentos com níveis menores de água salina evidenciaram uma possível maior absorção de nutrientes pelas plantas de feijoeiro. Esses mesmos autores relataram que o aumento dos níveis de salinidade da água de irrigação ocasiona um menor consumo de água pelas plantas em função do maior acúmulo de sais no solo, o que provoca uma menor absorção de nutrientes (Garcia et al., 2008).

\section{Considerações Finais}

O tratamento, água do sistema de ar condicionado (T2) demonstrou como alternativa viável para a utilização na irrigação de mudas de pinheira.

O tratamento T4 não é recomendável para irrigação de mudas pinheira de devido ao nível toxidez provocado pelos elevados teores de sódio e cloreto.

O tratamento T3 induziu o aumento do sódio e porcentagem de sódio trocável (PST) no solo.

\section{Referências}

Ayers RS \& Westcot DW. (1991). A qualidade da água na agricultura. 2.ed. Campina Grande: UFPB.

Bezerra DEL, Filho PL, Pereira Jr, EB, Azevedo PRE \& Silva EA. (2019). Mudas de mamoeiro (Carica Papaya L.) irrigadas com diferentes tipos de água. Revista Verde de Agroecologia e Desenvolvimento Sustentável. 14(1) jan.-mar.

Carmona, F. C., Anghinoni, I., \& WEBER, E. J. (2011). Salinidade da água e do solo e seus efeitos sobre o arroz irrigado no Rio Grande do Sul. - Cachoeirinha: IRGA/Estação Experimental, Seção de Agronomia.

Chartzoulakis, K., \& Klapaki, G. (2000). Response of two greenhouse pepper hybrids to $\mathrm{NaCl}$ salinity during diferente growth stages. Scientia Horticulturae, Amsterdam, v. 86, n. 3. 
Costa, D. M. A. DA., \& Barros Júnior, E A. C. de. (2005). Avaliação da necessidade do reuso de águas residuais. Holos, Ano 21, setembro.

Ferreira, D. F. (2011). SISVAR: Um sistema computacional de análise estatística. Revista Ciência e Agrotecnologia. Lavras/MG. v.35, n. 6, nov-dez.

Ferreira Neto, J., De Queirós, M. M. F., Nobre, R. G., Pereira Junior, E. B., Sousa, J. C., \& Sousa, J. X. (2017). Caracterização físico-química e microbiológica da beterraba irrigada com efluente agroindustrial. Rev. de Agroecologia no Semiárido, v. 1, n.1.

Garcia, G.O., Martins Filho, S., Reis, E. F., Moraes, W. B., \& Nazário, A. A. (2008). Alterações químicas de dois solos irrigados com água salina. Rev. Ciênc. Agron. Fortaleza, v. 39, n. 01, jan.- mar.

Gazeta, A. (2010). Anuário Brasileiro de Fruticultura. Gazeta: Santa Cruz do Sul.

Gurgel, M. T., Fernandes, P. D., Gheyi, H. R., Santos, F. J. S., \& Bezerra, I. L. (2007). Uso de águas salinas na produção de mudas enxertadas de aceroleira. Caatinga, v. 20, n. 2.

Herpin, U., Gloaguen, T. V., Fonseca, A. F., Montes, C. R., Mendonça, F. C., Piveli, R. P., Breulmann, G., Forti, M. C., \& Melfi, A. J. (2007). Chemicaleffectson the soil-plant system in a secondarytreatedwastewater irrigated coffee plantation - A pilotfieldstudy in Brazil. Agric. Water Manag.

Leal, R. M. P., Herpin, U., Fonseca, A. F., Firme, L. P., Montes, C. R., \& Melfi, A. J. Sodicityandsalinity in aBrazilianOxisolcultivated with sugarcane irrigated with wastewater. Agric. Water Manag., 96:307-316, 2009.

Janick, J. (1968). A ciência da horticultura. 485 p.

Lemos, E. E. P. (2014). A produção de anonáceas no Brasil. Revista Brasileira de Fruticultura, v. 36, edição especial. 
Lima, F. V. S., Casimiro, D. E., Pereira Junior, E. B., Sousa, P. S., \& Cassimiro, C. A. L. (2019). Análise morfológica de mudas de maracujazeiro irrigadas com diferentes tipos de água. Rev.Bras.de Gestão Ambiental (Pombal, PB), 13(02), abr./jun.

Leonel, S., Araújo, J. F., \& Tecchio, M. A. (2015). Biofertilização e adubação organomineral: concentração de nutrientes na folha e produtividade de frutos de pinheira. Irriga, Botucatu, Edição Especial, 20 anos Irriga + 50 anos FCA.

Marchal, J., \& Bertin, Y. (1980). Contenu en elements minéraux des organs de lavocatier "hula" at relations avec la fumure. Fruits, 35 (3).

Minami, K., et al. (1994). Produção de mudas hortícolas de alta qualidade. Piracicaba ESALQ/SEBRAE.

Nóbrega, E. P., Sarmento, M. I. A., Rodrigues, M. L. M., Oliveira, P. R. R., Neto, J. F., \& Maracajá, P. (2018). Desenvolvimento inicial de mudas de goiabeira irrigadas com diferentes tipos de água. Revista de Agroecologia no Semiárido, [S.1.], v. 1, n. 2, p. 01-09.

Pereira Jr, E. B., Sousa, J. F., Lima, C. J., Hafle, O. M., Gomes, D. J., \& Oliveira, F. T. (2018). Quantidade e qualidade da água dos aparelhos condicionadores de ar no IFPB Campus Sousa, PB. Revista de Agroecologia no Semiárido, v. 1, n. 1.

Prado, R. de M. et al. (2003). Resposta de mudas de goiabeira à aplicação de escória de siderurgia como corretivo de acidez do solo. Revista Brasileira de Fruticultura, Jaboticabal, v. 25 , n. 1.

Pushard, T. S. (2008). The State of Rainwater Harvesting. In The U.S. On Tap.

Santos, F. J. de S. (2000). Produção de mudas de pé franco de graviola (Annona muricata L.) irrigadas com água de diferentes qualidades. In: CONGRESSO BRASILEIRO DE ENGENHARIA AGRÍCOLA, 29., 2000, Fortaleza. Anais... Fortaleza: Sociedade Brasileira de Engenharia Agrícola/UFC. 
Sá, F. V. S., Pereira, F. H. F., Lacerda, F. H. D., \& Silva, A. B. da. (2013). Crescimento inicial e acúmulo de massa seca de cultivares de mamoeiro submetidas à salinidade da água em cultivo hidropônico. Revista Brasileira de Ciências Agrárias, v.8, n.3.

Sá, F. V. S., Brito, M. E. B., Ferreira, I. B., Antônio Neto, P., Silva, L. A., \& Costa, F. B. (2015). Balanço de sais e crescimento inicial de mudas de pinheira (Annona squamosa L.) sob substratos irrigados com água salina. Irriga, Botucatu, v. 20, n. 3.

Santana, M. J. et al. (2003). Efeito da irrigação com água salina em um solo cultivado com feijoeiro (Phaseolus vulgarisL.). Ciência e Agrotecnologia. Lavras, v. 27, n. 02.

Silva, D. A. (1997). Efeitos de fontes e níveis de salinidade sobre a germinação e desenvolvimento de plântulas de graviola (Annona muricata L.). 1997. 64 f. Dissertação (Mestrado em Manejo de Solos) - Universidade Federal da Paraíba, Areia.

Távora FJAF, Lima ECC \& Hernandez FFF. (2004). Composição mineral das raízes caules e folhas em plantas jovens de graviola submetidas a estresse salino. Ciência Agronômica, Fortaleza, 35(1).

Wagner Jr A, Neres L, Rodrigo C, Silva N, Rondinelli J, Rodrigo SA, Diniz ER, Duarte PL \& Claudio HB. (2016). Substratos na formação de mudas de pinheira (Annona squamosa L.). Revista Ceres, 53(308), julho-agosto.

\title{
Porcentagem de contribuição de cada autor no manuscrito
}

\author{
Edvanildo de Andrade - 12\% \\ Maria Iza de Arruda Sarmento - 12\% \\ Ednaldo Barbosa Pereira Junior - 12\% \\ Joserlan Nonato Moreira - 12\% \\ Éverson Pedrosa da Nóbrega - 12\% \\ Clarice Ribeiro Alves Caiana - 10\% \\ Francisco das Chagas Bezerra Neto - $10 \%$ \\ Aline Carla de Medeiros - 10\% \\ Patrício Borges Maracajá - 10\%
}

